\title{
miR-186 functions as a tumor suppressor in osteosarcoma cells by suppressing the malignant phenotype and aerobic glycolysis
}

\author{
QIANREN XIAO $^{1,3^{*}}$, ZHIHUI WEI $^{1 *}$, YUNYUN LI $^{2 *}$, XIN ZHOU $^{1}$, JIAJUN CHEN $^{1}$, \\ TENGYU WANG ${ }^{1}$, GAOHAI SHAO ${ }^{1}$, MINGHUA ZHANG ${ }^{1}$ and ZHONGZU ZHANG $^{1}$ \\ Departments of ${ }^{1}$ Orthopedics and ${ }^{2}$ Gynecology, The Yongchuan Hospital of Chongqing Medical University, \\ Chongqing 402160; ${ }^{3}$ Department of Pain Management, \\ The Affiliated Hospital of Jinggangshan University, Ji'an, Jiangxi 343000, P.R. China
}

Received November 17, 2017; Accepted January 12, 2018

DOI: $10.3892 /$ or.2018.6394

\begin{abstract}
Osteosarcoma (OS) is the most common primary bone malignancy among children and adolescents. Deregulation of microRNAs has been well documented in OS, while the putative effects of miR-186 have not been identified yet. In the present study, we assessed the expression of miR-186 in a cohort of 40 OS tissues and explored its effects on OS cells. As expected, miR-186 was suppressed in OS tissues compared with relative normal tissues. Overexpression of miR-186 inhibited cell proliferation, arrested the cell cycle progression and suppressed the cell invasion of the HOS and U2 OS cell lines. These results indicated the tumor-suppressive role of miR-186 in OS. Among the target genes of miR-186, we found that pituitary tumor transforming gene 1 (PTTG1) may be a target gene of miR-186 in OS and that the overexpression of PTTG1 could partially abolish miR-186-mediated suppressive effects on OS cells. Aerobic glycolysis is the major way of energy supply and is one of the characteristic phenotypes of tumor cells. In addition, we found that overexpression of miR-186 significantly suppressed the expression of hypoxia-inducible factor 1 (HIF-1) and inhibited the glucose uptake and lactate production of OS cells. Collectively, our findings demonstrated that miR-186 functions as a tumor suppressor in OS cells partially by targeting PTTG1 and that HIF-1-mediated suppression of aerobic glycolysis may be also involved in its suppressive effects.
\end{abstract}

Correspondence to: Dr Zhongzu Zhang, Department of Orthopedics, The Yongchuan Hospital of Chongqing Medical University, 439 Xuanhua Road, Yongchuan, Chongqing 402160, P.R. China

E-mail: zhangzhongzu@126.com

*Contributed equally

Key words: osteosarcoma, miR-186, PTTG1, malignant phenotype, aerobic glycolysis

\section{Introduction}

MicroRNAs (miRNAs) are a type of small ( 22-nt long), non-coding RNA molecules that regulate target gene expression by translational inhibition or mRNA destabilization through the combination with the 3'-untranslated region of target messenger RNAs. The loss of homeostasis in the miRNA/mRNA axis leads to a number of physiological and pathological events, such as inflammation, senescence and tumorigenesis $(1,2)$.

Osteosarcoma (OS) is the most common primary bone malignancy among children and adolescents (3). The exact pathological mechanisms of this malignancy remain unknown. Acting as the majority of non-coding RNAs, the effects of miRNAs in OS have been widely reported (4). For example, miR-224 was reported to be suppressed in OS tissues and was correlated with shorter survival time of OS patients. Overexpression of miR-224 promoted the sensitivity of OS cells to cisplatin by targeting Rac1 (5). In addition, our previous study reported that miR-140 was downregulated in OS tissues and functioned as a tumor suppressor in OS, as overexpression of miR-140 significantly inhibited cell proliferation in vitro and tumor growth in vivo by targeting the HDAC4 signaling pathway (6). Furthermore, miR-186 has been reported to function as a tumor suppressor in a variety of malignancies and regulate the chemosensitivity of non-small cell lung cancer cells to paclitaxel by targeting the MAPT-signaling pathway (7). In idiopathic pulmonary fibrosis cells, it has been closely related with the overexpression of collagen $\mathrm{V}$ and epithelial-to-mesenchymal transition (EMT) (8). However, its exact function in OS has not been identified yet. In the present study, the expression of miR-186 and its putative biological functions in OS cells were investigated.

\section{Materials and methods}

Clinical samples and cell culture. To explore the expression of miR-186 in OS, a cohort of 40 OS cases was adopted into the experiment. The detailed information of these patients are displayed in Table I. All patients had not followed any therapy before their recruitment to this study with written consents 
for their participation in this study. The present study was approved by The Institutional Ethics Committee of Chongqing Medical University. Adjacent normal tissues were collected from the patients as negative controls.

Human OS cell lines, U2 and HOS, were adopted for further biological function assays. The cell lines were both purchased from the Cell Bank of the Chinese Academy of Medical Sciences (Shanghai, China). The cells were all cultured in RPMI-1640 medium (Gibco, Grand Island, NY, USA) with $10 \%$ fetal bovine serum (FBS; Gibco) and incubated in a humidified atmosphere of $5 \% \mathrm{CO}_{2}$ at $37^{\circ} \mathrm{C}$.

Oligonucleotides and cell transfection. MicroRNA-186 and relative scramble mimic oligonucleotides were all purchased from Dharmacon (Lafayette, CO, USA). A final concentration of $50 \mathrm{nM}$ mimics was transfected into OS cells using the DhamaFECT 1 transfection reagents according to the manufacturer's instructions. After 6-h transfection, the medium was changed and the cells were cultured for $48 \mathrm{~h}$ and harvested for further experiments.

$R N A$ extraction and $q R T-P C R$. To assess the expression level of miR-186, quantitative real-time PCR (qRT-PCR) was performed using SYBR Green (TransGen Biotech, Beijing, China) in the Bio-Rad real-time PCR system. Total RNA was extracted from the cells and tissues using TRIzol (Invitrogen Life Technologies, Carlsbad, CA, USA) according to the manufacturer's instructions. The data of miR-186 and PTTG1 expression were normalized to endogenous U6 snRNA and GAPDH, respectively. The primer for reverse transcription was used as follows: 5'-GTCGTATCCAGTGCAGGGTCC GAGGTATTCGCACTGGAGCCCAA-3'. The forward and reverse primers for miR-186 RT-PCR were 5'-CGCGGCAAA GAATTCTCCTT-3' and 5'-GCCGCAGTGGTTTTACCCT-3', respectively. The forward and reverse primers for PTTG1 were 5'-TTTGACCTGCCTGAAGAGC-3' and 5'-CGACAGAAT GCTTGAAGGAG-3' respectively. The forward and reverse primers for U6 and GAPDH were used as follows: For U6 5'-CTCGCTTCGGCAGCACATATACT-3' and 5-ACGCTT CACGAATTTGCGTGTC-3', respectively and for GAPDH, 5'-CTCGCTTCGGCAGCACATATACT-3' and 5'-ACGCTT CACGAATTTGCGTGTC-3', respectively. Subsequently, the PCR data were analyzed using the $2^{-\Delta \Delta C T}$ method.

Luciferase reporter assay and vector construction. To identify the direct target role of PTTG1 on miR-186 by connecting with its 3'-UTR, the Dual-Luciferase Reporter assays were adopted. The full-length 3'-UTR of the PTTG1 mRNA was amplified from genomic DNA and then cloned into the pGL-3 vector (Promega, Madison, WI, USA). Mutations of PTTG1 3'-UTR sequence were created through the QuickChange Site-Directed Mutagenesis kit according to the manufacturer's instructions (Stratagene; Agilent Technologies, Inc., Santa Clara, CA, USA). Before transfection, about $1 \times 10^{5}$ cells/well were seeded into 24 -well plates for $24 \mathrm{~h}$. The cells were transfected with $10 \mathrm{ng}$ pRL-TK Renilla Luciferase reporter, $50 \mathrm{ng}$ pGL-3 firefly Luciferase reporter and $50 \mathrm{nM}$ miRNA-186/scramble mimic. The pRL-TK vector served as internal control and the luciferase reporter construct containing the miRNA-186 consensus target sequence served as positive control. All transfections were carried out with Lipofectamine 2000 (Invitrogen Life Technologies). At $48 \mathrm{~h}$ after transfection, the cells were collected and lysed using the Passive Lysis Buffer (Promega). Finally, the Luciferase activity was assessed using the Dual-Luciferase Reporter assay (Promega). The results were normalized to the Renilla Luciferase.

Cell proliferation assay. The effects of miR-186 on the cell proliferation rate of OS cells were assessed using Cell Counting Kit-8 (CCK-8) assays. At $24 \mathrm{~h}$ after transfection $0.5 \times 10^{4}$ HOS or U2 cells were seeded into the 96-well plates. The cells were incubated in 10\% CCK-8 (Dojindo Molecular Technologies, Inc., Kumamoto, Japan) and diluted in normal culture medium at $37^{\circ} \mathrm{C}$ until visual color conversion occurred. The proliferation rate was determined at $0,24,48$ and $72 \mathrm{~h}$ after the cells were seeded into 96 -well plates and quantification was performed on a microtiter plate reader according to the manufacturer's instructions. All experiments were performed in triplicate.

Cell cycle and apoptosis analysis. For the analysis of the cell cycle, HOS or U2 cells were harvested, diluted to a concentration of $5 \times 10^{5}$ cells $/ \mathrm{ml}$ and washed twice with ice-cold PBS $48 \mathrm{~h}$ after transfection. Then the cells were fixed in ice-cold $70 \%$ ethanol and incubated with propidium iodide (PI) and RNase A. For the analysis of apoptosis, the cells were incubated with PE Annexin V and 7-AAD according to the PE Annexin V Apoptosis Detection kit I (BD Biosciences, San Jose, CA, USA) protocol. All cells in both assays were then analyzed by fluorescence-activated cell sorting (FACS). Cells that bound to PE Annexin V underwent early apoptosis, while cells that bound to both PE Annexin V and 7-AAD were either in the late stages of apoptosis or already dead. All experiments were run in triplicate.

Cell Matrigel assay. For analyzing the effects of miR-186 on cell migration and invasion, the Matrigel chamber assays were adopted. After a 24 -h transfection, $2 \times 10^{5}$ HOS or U2 cells suspended in serum-free medium were added to the upper chamber $(8-\mu \mathrm{m}$ pore filter). Medium containing $20 \%$ FBS was added to the lower chamber as a chemoattractant. For the invasion assays, the Transwell chambers were coated with Matrigel (BD Biosciences) and then incubated at $37^{\circ} \mathrm{C}$ for $4 \mathrm{~h}$ and inserted in 2x4-well plates. After incubation for $24 \mathrm{~h}$, the non-invasive cells on the upper surface of the chamber were gently removed with a cotton swab. The cells passing through the chambers and located on the lower surface were stained with $0.05 \%$ crystal violet, air dried and photographed. All experiments were performed in triplicate.

Immunoblot assays. After $48 \mathrm{~h}$ transfection, $\mathrm{HOS}$ and $\mathrm{U} 2$ cells were harvested in PBS at $4^{\circ} \mathrm{C}$ and lysed on ice in cold-modified radioimmunoprecipitation buffer supplemented with protease inhibitors. Protein concentration was assessed using the BCA Protein Assay kit (Beyotime Institute of Biotechnology, Haimen, China) and equal amounts of protein were analyzed by SDS-PAGE. The gels were electroblotted onto nitrocellulose membranes (EMD Millipore, Billerica, MA, USA). The membranes were blocked for $1 \mathrm{~h}$ with $5 \%$ fat-free dry 
Table I. Clinicopathological factors of 40 OS patients.

\begin{tabular}{lc} 
Clinicopathological factors & No. of patients \\
\hline Sex & 23 \\
Male & 17 \\
Female & \\
Age (years) & 18 \\
$>25$ & 22 \\
$<25$ & \\
Tumor sites & 16 \\
Femur & 9 \\
Tibiofibules & 15 \\
Other sites & \\
Pathological types & 18 \\
Osteoblastic & 6 \\
Chondroblastic & 9 \\
Fibroblastic & 7 \\
Other types & \\
Alkaline phosphatase & 24 \\
Normal & 16 \\
Elevated & \\
\hline
\end{tabular}

milk in Tris-buffered saline containing $0.1 \%$ Tween-20 and incubated at $4^{\circ} \mathrm{C}$ overnight with primary antibody. Detection was assessed using peroxidase-conjugated secondary antibodies (1:1,000; anti-rabbit IgG; cat. no. 7054; Cell Signaling Technology, Inc., Danvers, MA, USA) and the enhanced chemiluminescence system (ECL; EMD Millipore). The primary antibodies used were PTTG1 $(1: 1,000$; rabbit, monoclonal; cat. no. 13445S), HIF-1 (1:1,000; rabbit, polyclonal; cat. no. 3716S) and GAPDH (1:1,000; rabbit, monoclonal; cat no. 5174S; Cell Signaling Technology). The experiments were performed three times.

Cell glucose uptake and lactate production assays. For the analysis of the glucose uptake, the Glucose Test kit (BioVision, Milpitas, CA, USA) was used. After transfection, HOS and U2 cells were seeded into 6 -well plates at a density of $10^{6}$ cells/ well at $37^{\circ} \mathrm{C}$ for $48 \mathrm{~h}$. The medium at $0 \mathrm{~h}$ was collected as background glucose concentration. The glucose concentration reduction of the medium was considered as cellular glucose uptake. Thus, glucose uptake was calculated as follows: glucose uptake $=$ (background concentration-reading concentration)/protein concentration. For assessing the extracellular lactic acid production, the culture medium of cells was explored using the Lactate Assay kit (BioVision) according to the manufacturer's instructions. The values were normalized to the protein concentration.

Statistical analysis. All data were derived from no less than three independent experiments, and are presented as the mean \pm SD. Statistical analysis was carried out using SPSS 18.0 software (IBM, New York, NY, USA). The Student's t-test was used for comparisons between two groups. $\mathrm{P} \leq 0.05$ was considered to indicate a statistically significant difference.

\section{Results}

miR-186 is suppressed in OS tissues. To explore the effects of miR-186 in OS, the expression level of miR-186 was detected in a cohort of 40 OS tissues and adjacent normal tissues through quantitative RT-PCR assays. Compared to the adjacent normal tissues, 30 out of 40 (75\%) OS tissues exhibited lower level of miR-186 expression (Fig. 1A). To further identify the expression tendency of miR-186 in OS, we analyzed its average level between OS tissues and normal tissues. As expected, the average level of miR-186 was significantly attenuated in OS tissues (Fig. 1B). The suppression of miR-186 in OS indicated its tumor-suppressive role in OS.

Overexpression of miR-186 inhibits cell proliferation and promotes cell apoptosis in OS cells. To identify the biological function of miR-186 in OS, the overexpression model of OS cells was established through transiently transfecting miR-186 mimic into the OS cell lines, HOS and U2. The cells transfected with scramble mimic were used as negative control. Upon transfection, the expression of miR-186 was $\sim 15$-fold higher in HOS cells (designed as HOS/186) and 18-fold higher in $\mathrm{U} 2$ cells (designed as U2/186) compared with control groups (Fig. 2A).

Subsequently, we explored the effects of miR-186 on the proliferation, cell cycle progression and apoptosis of OS cells. The rate of cell proliferation was assessed in both cell lines in vitro using the CCK-8 assay. The doubling time of HOS/186 and U2/186 cells was significant lower than that of HOS/Scr and U2/Scr cells, indicating that overexpression of miR-186 inhibited the growth rate of OS cells (Fig. 2B). Further FACS assays revealed that overexpression of miR-186 resulted in a significant upregulation of early apoptotic cells (Fig. 2C), associated with a simultaneous increased amount of cells in the G1-phase and a reduced number of cells in the S-phase of the cell cycle (Fig. 2D), which indicated that overexpression of miR-186 inhibited cell proliferation and induced cell apoptosis of OS cells.

Overexpression of miR-186 suppresses cell invasion in OS cells. Tumor metastasis is a pivotal reason for the poor clinical outcome of OS. Using neoadjuvant chemotherapy with surgery, the five-year survival rate of patients with non-metastatic OS has increased $>50 \%$, while the prognosis of patients with tumor metastasis is still poor (9). Thus, we further explored the effects miR-186 on cell invasion of OS cells using the Matrigel chamber assays. The OS cells transfected with miR-186 (HOS/186 and U2/186 cells) exhibited less cells passing through the chambers coated with Matrigel (Fig. 2E), which means that transfection with miR-186 significantly suppressed the invasive capacity of OS cells.

miR-186 modulates the expression of PTTG1 in OS cells. The aforementioned data strongly indicated that miR-186 functioned as a tumor suppressor in OS cells. To elucidate the putative mechanisms involved in miR-186-mediated tumor suppressive effects in OS, we investigated its target genes using the target prediction software, miRanda (http://www. targetscan.org/vert_71/) and TargetScan (http://34.236.212.39/ microrna/microrna/home.do). Both these tools indicated that 

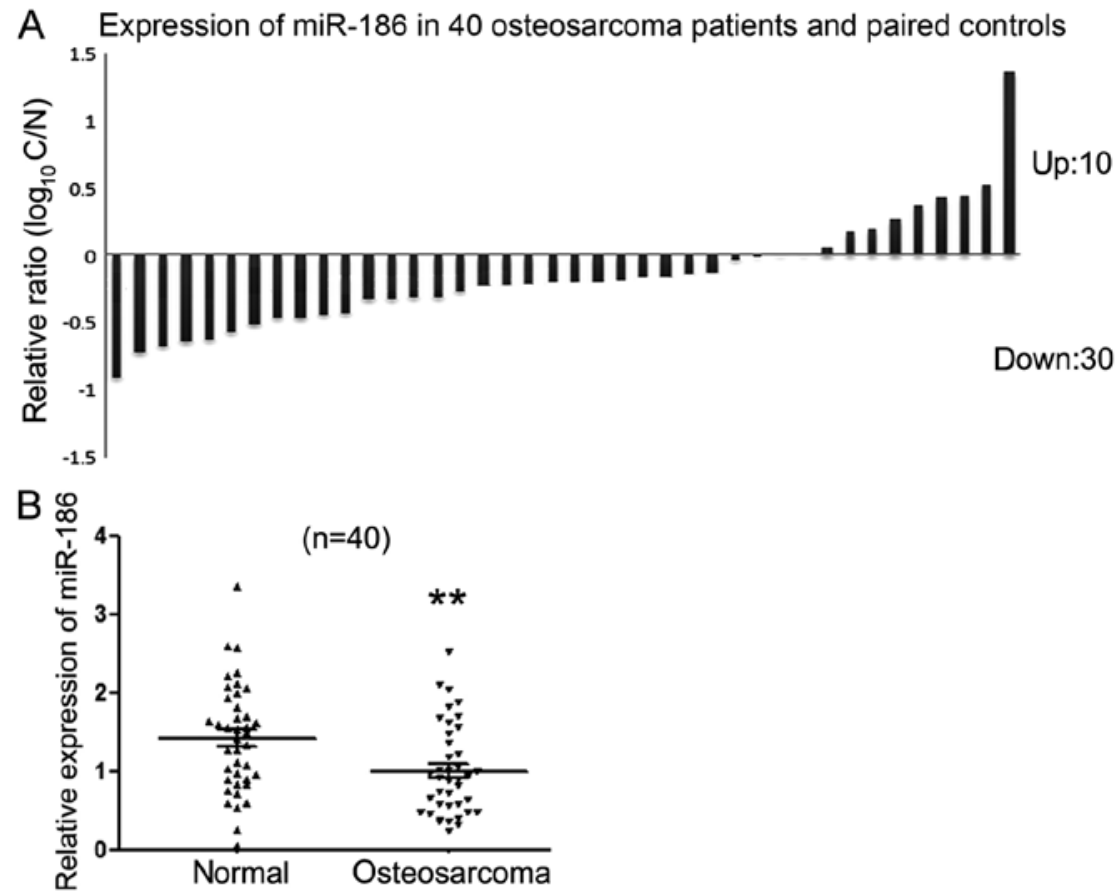

Figure 1. miR-186 is suppressed in OS tissues. (A) The expression of miR-186 was detected in 40 OS patients and paired controls using the quantitative RT-PCR. Compared with the normal tissues, $75 \%$ of the patients demonstrated downregulation of miR-186. (B) The relative mean level of miR-186 in OS tissues and adjacent normal tissues was explored using statistical analysis. The mean level of miR-186 in OS tissues was much lower. ${ }^{* *} \mathrm{P}<0.01$.
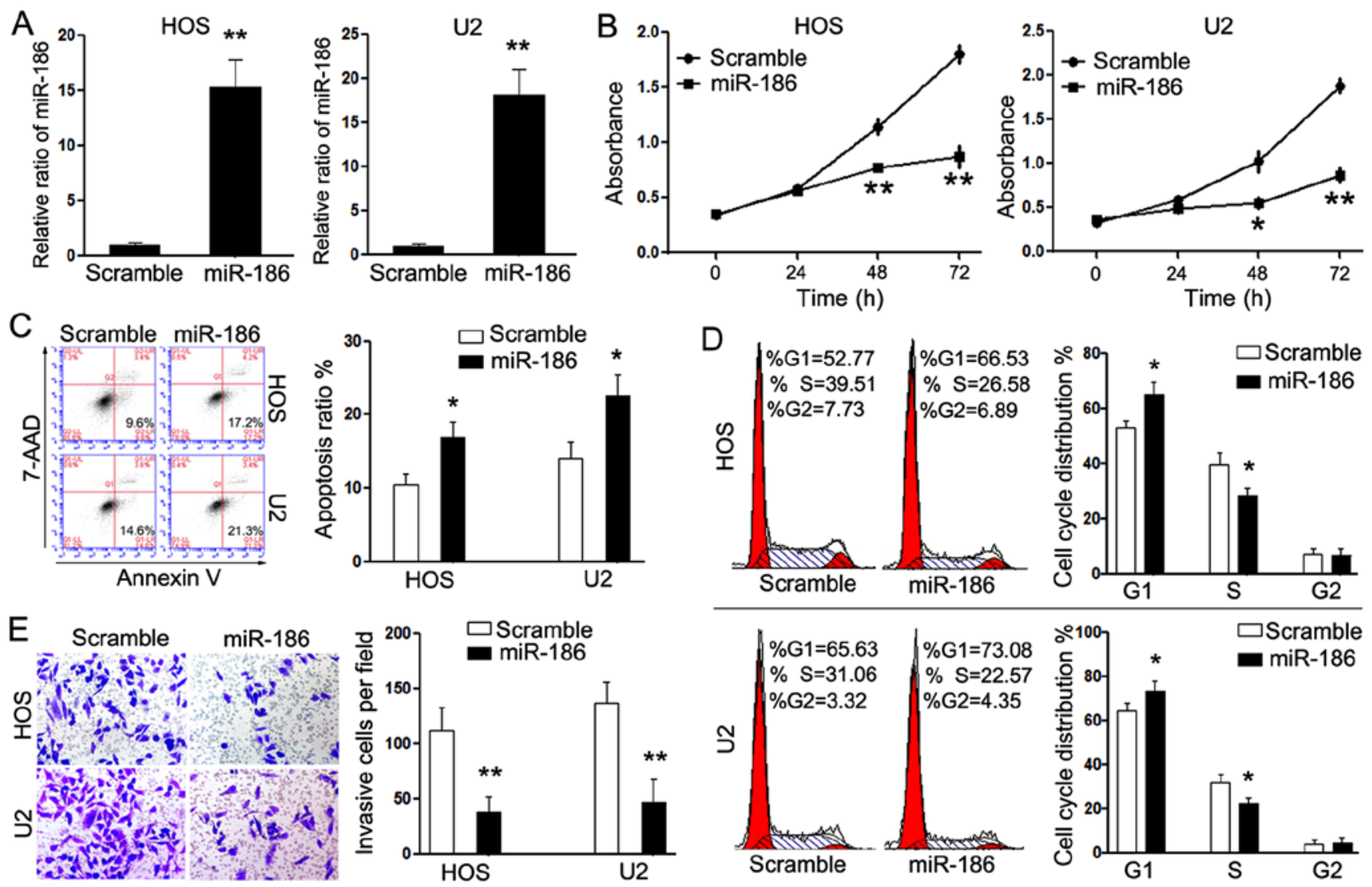

Figure 2. miR-186 inhibits cell proliferation and invasion of OS cells and promotes cell apoptosis. (A) HOS and U2 cells were transfected with miR-186 mimic for $48 \mathrm{~h}$, and then the expression of miR-186 was detected using the qRT-PCR analysis. Upon transfection, the expression of miR-186 was significantly increased. (B) CCK-8 assays were performed to explore the effects of miR-186 on the cellular viability of HOS and U2 cells. Overexpression of miR-186 suppressed cell proliferation of the HOS and U2 cells. Fluorescence activated cell sorting (FACS) analysis was performed to explore the effects of miR-186 on (C) cell apoptosis and (D) cell cycle progression of the HOS and U2 cells. Treatment with miR-186 induced early apoptosis and suppressed the cell cycle progression of HOS and U2 cells. (E) Matrigel chamber assays were performed to explore the effects of miR-186 on cell invasion of the OS cells. Restored expression of miR-186 reduced cells passing through the chambers coated with Matrigel (x100 magnification). " $\mathrm{P}<0.05 ;{ }^{* *} \mathrm{P}<0.01$. 

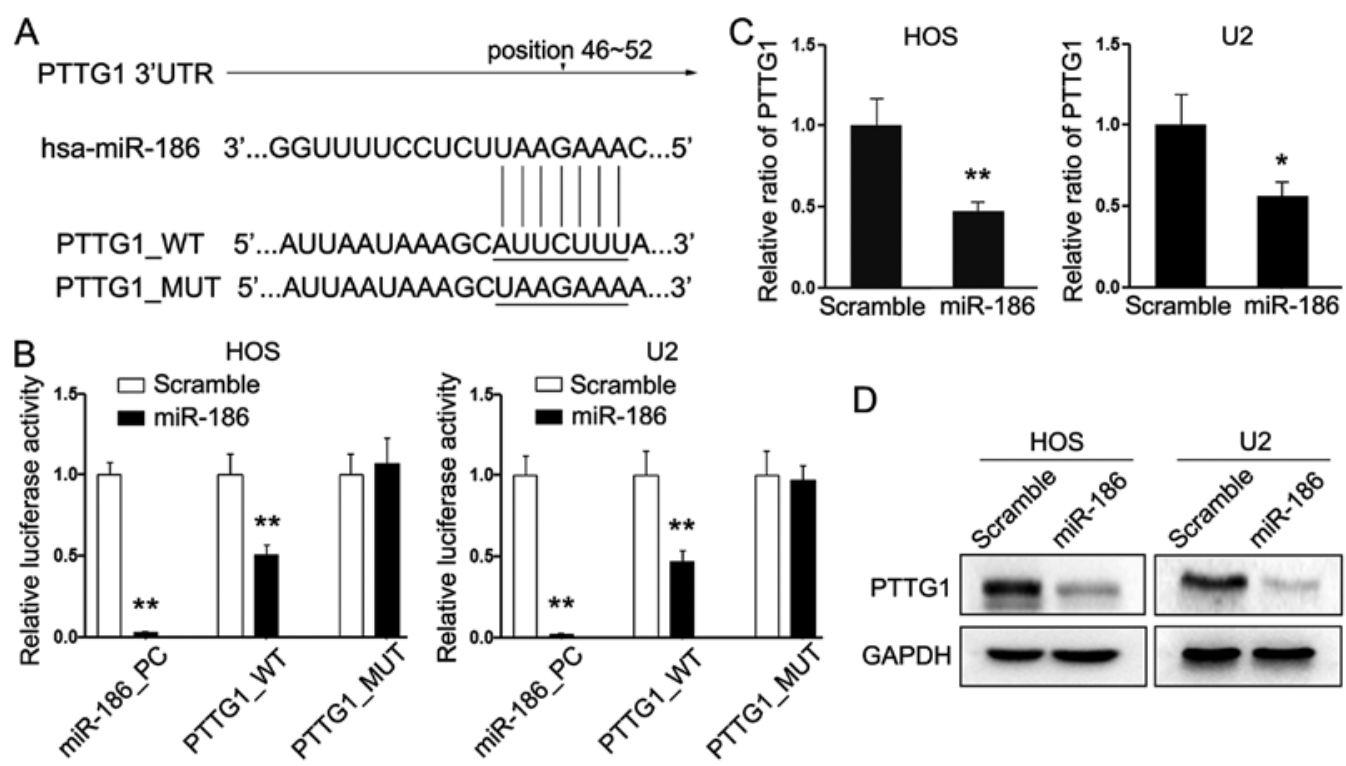

Figure 3. miR-186 directly targets PTTG1 in HOS and U2 cells. (A) Schematic representation of 3'UTR of PTTG1 gene revealing the putative binding site of miR-186. (B) Relative luciferase activity of the indicated PTTG1 reporter construct in HOS and U2 cells is demonstrated. miR-186 mimic suppressed the luciferase activity of the reporter gene containing wild-type 3'-UTR of PTTG1, but not the mutant one in HOS and U2 cells. (C) The expression of PTTG1 was detected in HOS and U2 cells upon transfection with miR-186. Exogenetic overexpression of miR-186 significantly decreased the PTTG1 transcripts (D) The expression of PTTG1 protein was examined using the immunoblot assays. The expression of PTTG1 was significantly suppressed upon transfection with miR-186. ${ }^{* *} \mathrm{P}<0.01 .{ }^{*} \mathrm{P}<0.05$.

PTTG1 was a target gene of miR-186 (Fig. 3A). PTTG1 has been reported to function as an oncogene in a cohort of cancers, including OS (10). Inhibition of PTTG1 in MG-63 cells caused cell cycle arrest and subsequent apoptosis (11). To identify the target role of PTTG1 in OS cells, the dual-luciferase reporter assays were adopted. Compared to the cells transfected with scramble mimic, cells treated with miR-186 mimic demonstrated significantly attenuated luciferase activity when co-transfected with the reporter gene containing wild-type 3'-UTR of PTTG1. However, transfection with miR-186 mimic did not affect the luciferase activity when co-transfected with gene containing the mutant 3 '-UTR in HOS and U2 cells (Fig. 3B). Furthermore, western blot analysis and qRT-PCR analysis confirmed that the PTTG1 protein as well as mRNA levels were indeed reduced drastically in HOS/186 and U2/186 cells consistently (Fig. 3C and D). Collectively, these data strongly indicated that PTTG1 is a target gene of miR-186 in OS cells.

PTTG1 is involved in miR-186-mediated suppressive effects. As aforementioned, PTTG1 functions as an oncogene in a cohort of cancer cells. We hypothesized that PTTG1 may be involved in the miR-186-mediated suppressive effects in OS cells. To validate this hypothesis, rescue assays were performed. The PTTG1/control constructs were transfected to the OS cells which had been previously transfected with miR-186/scramble mimic. As displayed in Fig. 4A, the expression of PTTG1 was partially restored upon transfection with PTTG1 constructs. Notably, accompanied with the restored expression of PTTG1, an increased cell proliferation (Fig. 4B) as well as suppressed cell apoptosis (Fig. 4C) were observed in OS cells transfected with PTTG1 constructs and miR-186 mimic. Furthermore, co-transfected with PTTG1 constructs also partially blocked miR-186-mediated suppression of invasion (Fig. 4D). All these results indicated that PTTG1 was involved in miR-186-mediated tumor suppressive effects in OS.

miR-186 suppresses the expression of HIF-l $\alpha$ and glycolysis of OS cells. Aerobic glycolysis is the major method of energy supply and thus, one of the characteristic phenotypes of tumor cells. It was reported that miR-186 significantly inhibited aerobic glycolysis in gastric cancer by suppressing hypoxia inducible factor $1 \alpha($ HIF-1 $\alpha)(12)$, which has been demonstrated to function as an oncogene in OS (6). Thus, we investigated whether HIF-1 $\alpha$-mediated dysregulation of aerobic glycolysis was also involved in the suppressive effects of miR-186. As expected, overexpression of miR-186 significantly suppressed the expression of HIF-1 $\alpha$ in HOS and U2 cells (Fig. 5A). Consistently, HOS and U2 cells that were treated with miR-186 had less intracellular glucose and lactate production (Fig. 5B and C). These results indicated that HIF-1 $\alpha$-regulated glycolysis may be involved in the miR-186-mediated suppressive effects on OS cells.

\section{Discussion}

Research on the current mechanisms underlying the progression of OS is still limited. Acting as one of the most important non-coding RNAs, miRNAs have been widely explored in the tumorigenesis and tumor progression of malignancies, including OS. miR-186 is a newly reported miRNA and its effects in OS remain unknown. In the present study, we assessed the expression of miR-186 in OS tissues and detected its biological functions on the OS cells and the regulatory mechanisms involved.

miR-186 is a conservative gene, which resides between exon 8 and 9 of the zinc finger RAN-binding domain containing 2 (ZRANB2) gene (13). Despite being found to 

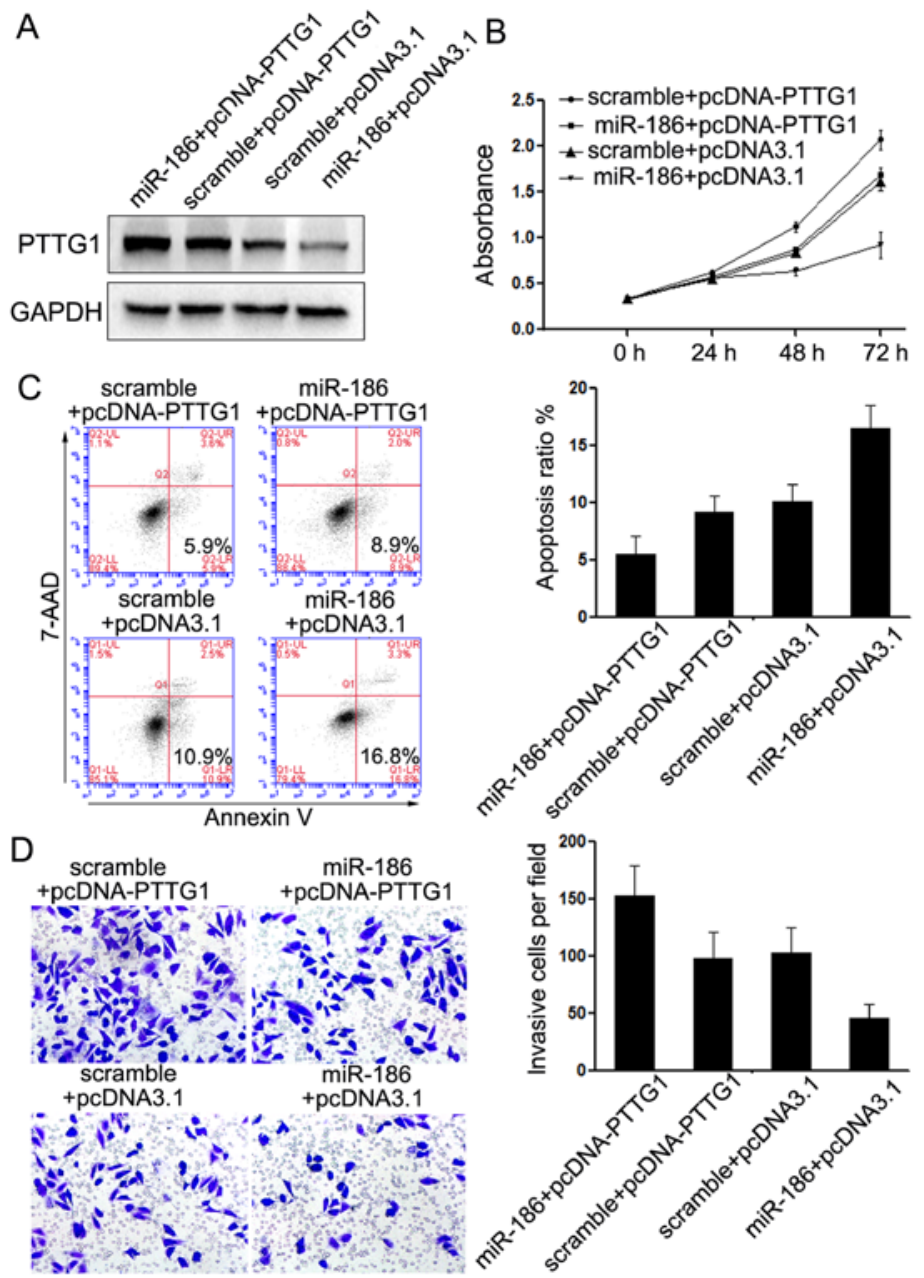

Figure 4. PTTG1 is involved in miR-186-mediated suppressive effects in OS cells. (A) The expression of PTTG1 was restored when PTTG1 constructs were transfected into HOS and U2 cells which were previously treated with miR-186 mimic. The expression of GAPDH was used as the control. (B) The cell proliferation rate mentioned in A, which were treated with miR-186/scramble mimic and PTTG1/control constructs, was detected using CCK-8 assays. Cells transfected with miR-186 and control constructs exhibited the lowest proliferation rates. (C) Cell apoptosis of HOS and U2 cells treated as described above was detected. The group transfected with miR-186 and control constructs exhibited the maximum number of early apoptotic cells, which could be abolished upon transfection with PTTG1 constructs. (D) Transwell assays were conducted to detect the effects on cell invasion of HOS and U2 cells. The group transfected with miR-186 and control constructs exhibited the minimum number of cells passing through the chamber, which could be abolished upon transfection with PTTG1 constructs. "P<0.05.

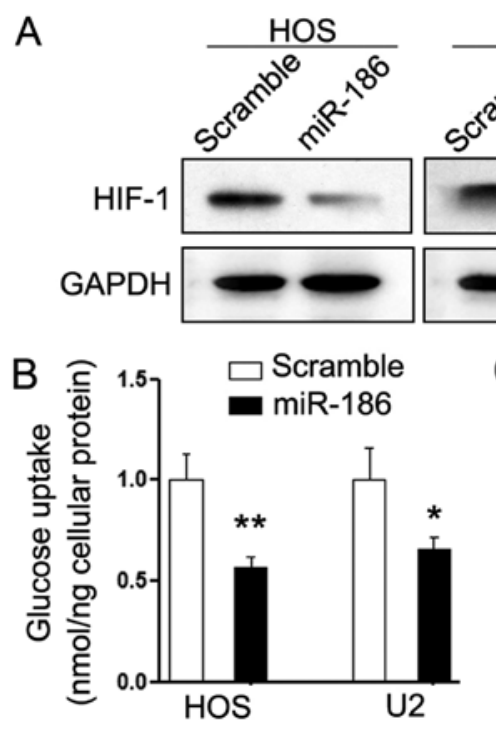

$\mathrm{U} 2$ 
perform a pivotal role in muscle differentiation (14), miR-186 has also been found to participate in many biological and pathological processes, including cell proliferation, apoptosis and invasion $(7,15)$. Recently, the tumor suppressive role of miR-186 has been widely reported in many cancers, such as in prostate cancer (PC), where miR-186 inhibited the expression of VEGF and reduced tumor growth by influencing the PGE-2-induced VEGF-signaling pathway (16). In addition, in gastric cancer cells, miR-186 inhibited cell proliferation, migration and invasion by targeting Twist1 (15). In the present study, we investigated the expression of miR-186 in 40 pairs of OS tissues and relative normal tissues. As expected, the expression of miR-186 was consistently suppressed in OS tissues and the restored expression of miR-186 inhibited cell proliferation, arrested cell cycle progression, induced cell apoptosis and inhibited cell invasion of OS cells. These results revealed the tumor-suppressive role of miR-186 in OS.

To investigate the mechanisms involved in miR-186-mediated suppressive effects, we searched for its target genes. Among the predicted genes, PTTG1 attracted most attention. PTTG1 is a newly identified oncogene and it is highly expressed in pituitary tumors and other neoplasms. The expression of PTTG1 is cell cycle-dependent; it peaks at the G2/M phase of the cell cycle and is degraded at the initiation of anaphase (13). Furthermore, overexpression of PTTG1 causes p53-dependent and p53-independent apoptosis and inhibits cell mitosis (11). In OS cells, overexpression of PTTG caused cell cycle arrest and subsequent apoptosis, while inhibition of PTTG promoted cell survival. Interestingly, OS cells expressing PTTG demonstrated signs of aneuploidy including the presence of micronuclei and multiple nuclei (11). The oncogenic role of PTTG1 in OS combined with the fact that PTTG1 was reported to be involved in miR-186-mediated tumor suppressive effects on non-small cell lung cancer cells led to the hypothesis that it may participate in miR-186-mediated effects on OS cells. Further experiments strongly supported our hypothesis as the rescued expression of PTTG1 in the OS cells which had been previously transfected with miR-186 partially abolished its suppressive effects on cell proliferation and invasion. In combination with previous research results, it suggested that PTTG1 partially participated in miR-186-mediated tumor suppressive effects.

A hypoxic microenvironment is common in many types of solid tumors, including OS $(17,18)$. Hypoxia may induce aerobic glycolysis, which is one of the characteristic phenotypes of malignant cells and is the major way of energy supply (12). HIF-1 $\alpha$ may be involved in this mechanism (19). We found that overexpression of miR-186 significantly suppressed the expression of HIF-1 in OS cells and inhibited glucose uptake and lactate production. These results indicated that HIF-1 $\alpha$-mediated dysregulation of aerobic glycolysis may be involved in the miR-186-mediated suppressive effects on OS cells and are partially consistent with our previous study that HIF-1 $\alpha$ acts as an oncogene in OS cells (12). However more studies are warranted in order to identify the underlying mechanisms that participate in the suppressive effects on aerobic glycolysis.

The findings of the present study strongly demonstrated that miR-186 functions as a tumor suppressor in OS cells through, at least partially, the suppression of the PTTG1-mediated oncogenic effects on the malignant phenotypes of OS cells and HIF-1-mediated suppression on aerobic glycolysis. To the best of our knowledge, this is the first comprehensive study to explore the role of miR-186 in OS. A combined miRNA-based and epigenetic treatment may be a novel potential therapeutic target for OS.

\section{Acknowledgements}

The present study was supported by the National Natural Science Foundation of China (grant no. 81502329), the Program of Science and Technology of Chongqing Commission (grant no. KJ1600228), the Programs of Yongchuan Hospital of Chongqing Medical University (grant nos. YJZQN 201514 and YCZQN 201511).

\section{Competing interests}

The authors declare that they have no competing interests.

\section{References}

1. Olivieri F, Capri M, Bonafè M, Morsiani C, Jung HJ, Spazzafumo L, Viña J and Suh Y: Circulating miRNAs and miRNA shuttles as biomarkers: Perspective trajectories of healthy and unhealthy aging. Mech Ageing Dev 165: 162-170, 2017.

2. Cao Q, Li YY, He WF, Zhang ZZ, Zhou Q, Liu X, Shen Y and Huang TT: Interplay between microRNAs and the STAT3 signaling pathway in human cancers. Physiol Genomics 45: 1206-1214, 2013

3. Kim HJ, Chalmers PN and Morris CD: Pediatric osteogenic sarcoma. Curr Opin Pediatr 22: 61-66, 2010.

4. Ram Kumar RM, Boro A and Fuchs B: Involvement and clinical aspects of MicroRNA in osteosarcoma. Int J Mol Sci 17: E877, 2016.

5. Geng S, Gu L, Ju F, Zhang H, Wang Y, Tang H, Bi Z and Yang C: MicroRNA-224 promotes the sensitivity of osteosarcoma cells to cisplatin by targeting Rac1. J Cell Mol Med 20: 1611-1619, 2016.

6. Xiao Q, Huang L, Zhang Z, Chen X, Luo J, Zhang Z, Chen S, Shu Y, Han Z and Cao K: Overexpression of miR-140 inhibits proliferation of osteosarcoma cells via suppression of histone deacetylase 4. Oncol Res 25: 267-275, 2017.

7. Ye J, Zhang Z, Sun L, Fang Y, Xu X and Zhou G: miR-186 regulates chemo-sensitivity to paclitaxel via targeting MAPT in non-small cell lung cancer (NSCLC). Mol Biosyst 12: 3417-3424, 2016.

8. Lei GS, Kline HL, Lee CH, Wilkes DS and Zhang C: Regulation of collagen $\mathrm{V}$ expression and epithelial-mesenchymal transition by miR-185 and miR-186 during idiopathic pulmonary fibrosis. Am J Pathol 186: 2310-2316, 2016.

9. Ando K, Heymann MF, Stresing V, Mori K, Rédini F and Heymann D: Current therapeutic strategies and novel approaches in osteosarcoma. Cancers (Basel) 5: 591-616, 2013.

10. Cai SQ, Dou TT, Li W, Li SQ, Chen JQ, Zhou J, Zheng M and Man XY: Involvement of pituitary tumor transforming gene 1 in psoriasis, seborrheic keratosis, and skin tumors. Discov Med 18: 289-299, 2014.

11. Yu R, Heaney AP, Lu W, Chen J and Melmed S: Pituitary tumor transforming gene causes aneuploidy and p53-dependent and p53-independent apoptosis. J Biol Chem 275: 36502-36505, 2000.

12. Liu L, Wang Y, Bai R, Yang K and Tian Z: miR-186 inhibited aerobic glycolysis in gastric cancer via HIF-1alpha regulation. Oncogenesis 6: e318, 2017.

13. Yu R, Ren SG, Horwitz GA, Wang Z and Melmed S: Pituitary tumor transforming gene (PTTG) regulates placental JEG-3 cell division and survival: Evidence from live cell imaging. Mol Endocrinol 14: 1137-1146, 2000.

14. Antoniou A,Mastroyiannopoulos NP, Uney JB and Phylactou LA miR-186 inhibits muscle cell differentiation through myogenin regulation. J Biol Chem 289: 3923-3935, 2014. 
15. Cao C, Sun D, Zhang L and Song L: miR-186 affects the proliferation, invasion and migration of human gastric cancer by inhibition of Twist1. Oncotarget 7: 79956-7996, 2016.

16. Terzuoli E, Donnini S, Finetti F, Nesi G, Villari D, Hanaka H, Radmark O, Giachetti A and Ziche M: Linking microsomal prostaglandin E Synthase-1/PGE-2 pathway with miR-15a and -186 expression: Novel mechanism of VEGF modulation in prostate cancer. Oncotarget 7: 44350-44364, 2016.

17. Koh MY, Spivak-Kroizman TR and Powis G: HIF-1alpha and cancer therapy. Recent Results Cancer Res 180: 15-34, 2010.
18. Masoud GN and Li W: HIF-1alpha pathway: Role, regulation and intervention for cancer therapy. Acta Pharm Sin B 5: 378-389, 2015.

19. Cheng SC, Quintin J, Cramer RA, Shepardson KM, Saeed S, Kumar V, Giamarellos-Bourboulis EJ, Martens JH, Rao NA, Aghajanirefah A, et al: mTOR- and HIF-1 $\alpha$-mediated aerobic glycolysis as metabolic basis for trained immunity. Science 345: 1250684,2014 